\title{
GESTIÓN DE RIESGO Y SU INFLUENCIA EN EL COMPORTAMIENTO RESILIENTE DE LOS ESTUDIANTES
}

\section{RISK MANAGEMENT AND ITS INFLUENCE ON THE RESILIENT BEHAVIOR OF STUDENTS}

\author{
Autora: Marjorie Abigail Tapia Bajaña \\ E-mail de contacto: magi leo @hotmail.com \\ ORCID ID: https://orcid.org/0000-0002-3835-9321 \\ Articulo recibido: 19 de Mayo del 2019 \\ Articulo revisado: 29 de Agosto del 2019 \\ Articulo aprobado: 01 de Diciembre del 2019 \\ Licenciada en Ciencias de la Educación mención Educadores de Parvulos egresada de la Universidad de Guayaquil \\ (Ecuador) con 12 años de experiencia en la docencia en la Escuela Particular "Semillita de Guayaquil. Maestrante en la \\ Universidad Nacional Mayor de San Marcos de Lima (Perú), mención Gestión de la Educación.
}

\section{Resumen}

La resiliencia ha recibido una mayor atención entre los profesionales y académicos en los últimos años. La resiliencia infantil ha recibido una atención notable en la reducción del riesgo de desastres (RRD) durante la creación del Marco de Sendai 2015-2030 para mejorar la protección de los niños en caso de desastres. Dado que la resiliencia es un concepto conceptual con una variedad de definiciones, este estudio evalúa sus diferentes factores y determina en la investigación existente para aclarar el camino para la investigación futura y objetiva. Se realizó una revisión sistemática de la literatura al buscar y seleccionar los artículos revisados por pares publicados en cuatro bases de datos electrónicas internacionales principales, que incluyen PubMed, SCOPUS, WEB OF SCIENCE y PsycINFO para responder a la pregunta de investigación. La mayoría de los estudios incluidos mencionaron los criterios dispersos sobre la resiliencia de los niños sin ninguna categoría organizada. Aunque este concepto es multifactorial, se necesita investigación adicional para desarrollar este estudio y también observar otros tipos de desastres, como los desastres provocados por el hombre.

\section{Palabras clave: seguridad, resiliencia, análisis de riesgos.}

\section{Abstract \\ Resilience has received greater attention among professionals and academics in recent}

years. Child resilience has received significant attention in disaster risk reduction (DRR) during the creation of the 2015-2030 Sendai Framework to improve the protection of children in the event of disasters. Since resilience is a conceptual concept with a variety of definitions, this study evaluates its different factors and determines in existing research to clear the way for future and objective research. A systematic review of the literature was performed by searching and selecting the peerreviewed articles published in four major international electronic databases, including PubMed, SCOPUS, WEB OF SCIENCE and PsycINFO to answer the research question. Most studies included scattered criteria about the resilience of children without any organized organization. Although this concept is multifactorial, additional research is needed to develop this study and also to observe other types of disasters, such as disasters caused by man.

Key words: security, resilience, risk analysis.

\section{Sumário}

A resiliência tem recebido maior atenção entre profissionais e acadêmicos nos últimos anos. A resiliência infantil recebeu atenção significativa na redução de riscos de desastres (RRD) durante a criação da Estrutura Sendai 2015-2030 para melhorar a proteção das crianças em caso de desastres. Como resiliência é um conceito conceitual com uma variedade 


\section{Ciencia y Educación (ISSN 2707-3378) \\ Vol. 1 No. 2 \\ Febrero del 2020}

de definições, este estudo avalia seus diferentes fatores e determina nas pesquisas existentes para esclarecer o caminho para pesquisas futuras e objetivas. Uma revisão sistemática da literatura foi realizada através da busca e seleção de artigos revisados por pares publicados em quatro grandes bancos de dados eletrônicos internacionais, incluindo PubMed, SCOPUS, WEB OF SCIENCE e PsycINFO para responder à pergunta da pesquisa. A maioria dos estudos incluídos mencionou os critérios dispersos sobre a resiliência das crianças sem nenhuma categoria organizada. Embora esse conceito seja multifatorial, são necessárias pesquisas adicionais para desenvolver este estudo e também observar outros tipos de desastres, como os causados pelo homem.

Palavras-chave: segurança, resiliência, análise de risco.

\section{Introducción}

La gestión de riesgo y su influencia en el comportamiento resiliente es un aspecto importante de la seguridad y protección debido a la mayor complejidad y conectividad de los sistemas y organizaciones dentro de la comunidad educativa. La seguridad es la "libertad de accidentes o pérdidas", (Academias G-Science, 2012). La resiliencia es "la capacidad de un sistema u organización para reaccionar y recuperarse de las perturbaciones en una etapa temprana, con un efecto mínimo en la estabilidad dinámica" (Foré \& Grané, 2010). Los accidentes e incidentes a menudo se deben a una combinación de vulnerabilidades. La capacidad de prever o recuperarse de accidentes e incidentes mejora tanto la seguridad como la protección. La resiliencia implica evitar y reducir las consecuencias de las perturbaciones desde una perspectiva de seguridad.

Existen sistemas resilientes que no son seguros; sin embargo, él objetivo es garantizar que los sistemas sean seguros y resistentes. Este estudio se centra en la gestión de riesgo y su influencia en el comportamiento resiliente los estudiantes, especialmente en los de octavo grado.

\section{Desarrollo}

Gestión del riesgo

El riesgo es la suma de posibles pérdidas que ocasionaría un desastre u otro evento adverso en términos de vida, condiciones de salud, medio de sustento, bienes y servicios, en una comunidad o sociedad particular en un período específico de tiempo en un futuro. Esta función de la amenaza-peligro y la vulnerabilidad, y es directamente proporcional a estos dos factores, por lo que se puede afirmar que el riesgo es dinámico y que puede aumentar o disminuir en la medida que ambos factores o uno de ellos varíen. La gestión de riesgo es un proceso social que considera las políticas nacionales para responder frente a los desastres.

"Es un proceso social cuyo fin último es la prevención, la reducción y el control permanente de los factores de riesgo de desastre en la sociedad, así como la adecuada preparación y respuesta ante situaciones de desastre, considerando las políticas nacionales, con especial énfasis en aquellas relativas a materia económica, ambiental, de seguridad, defensa nacional y territorial de manera sostenible. (Instituto Nacional de Defensa Civil, 2010, pág. 10)”

La gestión del riesgo de desastre está basada en la investigación científica y de registro de informaciones, y orienta las políticas, estrategias y acciones en todos los niveles de gobierno y de la sociedad con la finalidad de proteger la vida de la población y el patrimonio de las personas y del Estado. (Perú, 2011)

La gestión del riesgo parte del reconocimiento de que los desastres constituyan problemas generados en los procesos de desarrollo y por 


\section{Ciencia y Educación (ISSN 2707-3378) \\ Vol. 1 No. 2 \\ Febrero del 2020}

tanto la necesidad de fortalecer las capacidades y la articulación entre las diversas instituciones, organizaciones y los actores del desarrollo para reducir los riesgos.

Para Chuquisengo (2011, pág. 20) la gestión del riesgo es un proceso que contienen medidas y formas de intervención que tienden a prevenir los desastres siendo la capacidad de las sociedades y de sus actores sociales para transformar sus condiciones de riesgo, actuando sobre las causas que lo producen. Incluye medidas y formas de intervención que tienden a reducir, mitigar, prevenir y responder a los desastres. La transformación de las condiciones de riesgo debe darse a través de un proceso, planificado, concertado, participativo e integral una comunidad, una región o un país, ligado a la búsqueda de la sostenibilidad.

Para la presente investigación tomaremos la definición dada por el Centro Nacional de Estimación, Prevención y Reducción del Riesgo de Desastres (2014, pág. 12), mencionan que involucra las capacidades tanto nacionales, regionales y locales y se relaciona con el desarrollo sostenible:

Es el proceso planificado, concertado, participativo e integral de reducción de las condiciones de riesgo de desastres de una comunidad, una región o un país. Implica la complementariedad de capacidades y recursos locales, regionales y nacionales y está íntimamente ligada a la búsqueda del desarrollo sostenible.

La gestión de riesgo se debe transmitir conocimientos útiles a hombres, mujeres y niños sobre cómo prevenir o atenuar los impactos ocasionados por fenómenos naturales, por accidentes o errores de seguridad en cualquier lugar a través de: Proporcionar información de los organismos especializados en tareas de prevención y mitigación de desastres; estimular la planificación de acciones de prevención, simulacros y evacuación en los centros de estudios; motivar la capacidad de organizar grupos de apoyo mutuo y voluntario que funcionen en caso de desastres. (Municipalidad de Guayaquil, 2011)

\section{Tipos de gestión para reducir el riesgo}

De acuerdo con un documento elaborado por el Ministerio de Educación (2009, págs. 21-22) existen tres tipos de gestión de riesgo:

a. La gestión correctiva, que se refiere a la adopción de medidas y acciones de manera anticipada para reducir las condiciones de riesgo ya existente. Se aplica en base a los análisis de riesgos y teniendo en cuenta la memoria histórica de los desastres. Busca fundamentalmente revertir o cambiar los procesos que construyen los riesgos.

b. La gestión prospectiva, que implica adoptar medidas y acciones en la planificación del desarrollo para evitar que se generen nuevas condiciones de riesgo. Se desarrolla en función del riesgo "aún no existente" y se concreta a través de regulaciones, inversiones públicas o privadas, planes de ordenamiento territorial, etc. hacer prospección implica analizar el riesgo a futuro para definir el nivel de riesgo aceptable. Y para que sea exitosa, se requiere un alto grado de voluntad política, compromiso social y conciencia pública.

Lavell (2003, págs. 21-59) indica que los siguientes mecanismos deben aplicarse y se deben reforzar mutuamente:

1. La introducción de normativas y metodologías que garanticen que todo proyecto de inversión analice 
integralmente los riesgos que enfrenta y genera.

2. La creación de normativas sobre el ordenamiento territorial.

3. La búsqueda de usos productivos alternativos para territorios peligrosos.

4. La promoción de tecnologías accesibles y seguras, mediante normativas y programas.

5. El fortalecimiento de los niveles locales de gobierno, fundamentalmente de sus capacidades.

6. La continua sensibilización y capacitación sobre gestión de riesgos, teniendo en cuenta las particularidades de cada actor (sociedad civil, entes privados y estatales, cooperación internacional, etc.)

7. El establecimiento de penas y estímulos eficaces.

8. La instrumentación de esquemas de uso de recursos naturales y ecosistemas que garanticen el desarrollo sostenible.

9. La reforma del currículo.

10. El fomento de una cultura de seguridad.

11. La creación de espacios de participación ciudadana y de vínculos más estrechos entre los tomadores de decisiones y los actores locales.

c. La gestión reactiva que implica la preparación y la respuesta a emergencias, estando siempre alertados y bien preparados para cualquier eventualidad, de tal modo que los costos asociados a las emergencias sean menores, se presente un cuadro de daños reducido y la resiliencia sea alta.

Hasta ahora la mayor parte de políticas estatales en todo el mundo han dado más énfasis a la respuesta ante emergencias. El inconveniente de persistir de una política sesgada a esta fase de la gestión de riesgos es que favorece el asistencialismo más no el desarrollo y además sólo logra un alivio temporal.

También es usual que exista mucho apoyo para los procesos de rehabilitación después de desastres. En realidad, si dichos procesos están bien concebidos, representan una oportunidad para el fortalecimiento de las organizaciones locales y para fomentar un ordenamiento territorial más adecuado. La reconstrucción va ligada a la rehabilitación pues tiene fines similares pero su valor agregado es que pretende eliminar o reducir al máximo las limitaciones existentes antes del desastre, y también prevenir la generación de nuevos riesgos.

\section{Comportamiento resiliente}

La resiliencia se ha definido como la capacidad de una persona o grupo para seguir proyectándose en el futuro a pesar de acontecimientos desestabilizadores, de condiciones de vida difíciles y de traumas a veces graves (Manciaux, Vanistendael, Lecomte, \& Cyrulnik, 2003).

La resiliencia puede definirse como la capacidad de anticiparse frente a grandes desastres:

"La habilidad que tiene un sistema y sus componentes para anticipar, amortiguar, adaptar o recuperarse de los efectos de un desastre mayor, de forma oportuna $y$ eficaz. La capacidad de resiliencia debe desarrollarse en las instituciones a todos los niveles y sectores de la sociedad. (Academias G-Science, 2012, pág. 2)”

Actualmente, se hace referencia a la resiliencia como aquellas posiciones que toma una persona frente a los desastres:

"Las fortalezas que desarrollan y/o potencian las personas, familias y/o comunidades ante adversidades crónicas 
(ej. situaciones de pobreza, disfunción familiar...) o puntuales (ej. accidente, enfermedad, atentado terrorista, desastre natural...), siendo éstas, el resultado de los procesos que se generan de la interacción entre los factores de protección y de riesgo tanto individuales como ambientales. (Mateu, Gil, \& GarcíaRenedo, 2009)"

La resiliencia es la capacidad de los seres humanos para sobreponerse a las crisis y construir positivamente sobre ellas, basada en unos factores que facilitan y amplifican dicha respuesta. (Lamas, 2002) En otras palabras, es el sistema que se desarrolla por parte del individuo $o$ un grupo para enfrentar efectivamente la adversidad.

\section{Características del comportamiento resiliente}

De Foré y Grané (2010) se destaca las doce características principales de la resiliencia:

- Es un proceso

- Hace referencia a la interacción dinámica entre factores.

- Puede ser promovida a lo largo del ciclo de la vida.

- No se trata de un atributo estrictamente personal.

- Está vinculada al desarrollo y crecimiento humano.

- No constituye un estado definitivo.

- Nunca es absoluta ni total.

- Tiene que ver con los procesos de reconstrucción.

- Tiene como componente básico la dimensión comunitaria.

- Considera a la persona como única.

- Reconoce el valor de la imperfección

- Está relacionada con ver el vaso medio lleno.
Otras características del comportamiento resiliente para la presente investigación son los siguientes:

a. Manejo de actitudes emocionales. es la capacidad humana de sentir, entender, controlar y modificar estados emocionales en uno mismo y en los demás. La inteligencia emocional no es ahogar las emociones, sino dirigirlas y equilibrarlas. Empieza con la capacidad de reconocer los sentimientos y pensamientos propios, teniendo la sensibilidad y la empatía para identificar los ajenos y de esa manera dirigirlos y expresarlos sanamente. (Beltran, 2013)

b. Reducción de la Vulnerabilidad. es una condición previa que se manifiesta durante el desastre, cuando no se ha invertido suficiente en prevención $\mathrm{y}$ mitigación, y se ha aceptado un nivel de riesgo demasiado elevado. De aquí se desprende que la tarea prioritaria para definir una política preventiva es reducir la vulnerabilidad, pues no es posible enfrentarse a las fuerzas naturales con el objeto de anularlas. (Comisión Económica para América Latina y el Caribe, Banco Interamericano de Desarrollo, 2000)

Por otro lado, también se presentan características individuales como lo menciona García et. al. (2012, pág. 7):

a. Introspección. Hace mención a preguntarse a sí mismo y darse una auto respuesta honesta.

b. Independencia. Se define como la capacidad de establecer límites entre uno mismo y los ambientes adversos; alude a la capacidad de mantener distancia emocional y física, sin llegar a aislarse. 


\section{Ciencia y Educación (ISSN 2707-3378) \\ Vol. 1 No. 2 \\ Febrero del 2020}

c. La capacidad de relacionarse. Inciden en la habilidad para establecer lazos íntimos y satisfactorios con otras personas. Aquí encontraríamos cualidades como empatía, la sociabilidad.

d. Iniciativa. Implica exigirse y ponerse a prueba en tareas progresivamente más exigentes. Se refiere a la capacidad de hacerse cargo de los problemas y de ejercer control sobre ellos.

e. Humor. Alude al hecho de encontrar lo cómico en la tragedia. El humor ayuda a superar obstáculos y problemas, a hacer reír o reírse de lo absurdo de la vida.

f. Creatividad. Es la capacidad de crear orden, belleza y finalidad a partir del caos y el desorden. En la infancia se expresa con la creación de juegos, que son las vías para expresar la soledad, el miedo, la rabia y la desesperanza ante situaciones adversas.

g. Moralidad. Se refiere a la conciencia moral, a la capacidad de comprometerse de acuerdo a valores sociales y de discriminar entre lo bueno y lo malo.

Componentes para el desarrollo de la resiliencia

La adopción del enfoque de sistemas y la identificación de soluciones multidimensionales, son elementos clave para el desarrollo de la resiliencia. La Academia GScience (2012, págs. 3-5) define los siguientes cinco puntos para comprometer a la comunidad científica nacional e internacional en este esfuerzo:

a. Vigilancia continua de riesgos y evaluación periódica de desarrollo de capacidades. Es difícil estar preparados ante desastres que no logramos siquiera imaginar. Las regiones, los países y la comunidad internacional, deben desarrollar constantemente estrategias para la identificación y evaluación de los riesgos de desastres que enfrentan y reducir así su exposición. El monitoreo continuo es clave en este sentido.

b. Mejoramiento de los sistemas de salud pública. Incluso cuando un evento en curso no tiene que ver con los servicios de salud pública, las grandes crisis de índole social pueden rápidamente dar paso a una gran variedad de riesgos, incluso brotes de epidemias. Los sistemas de salud pública deben ser fortalecidos y mantenerse, tanto para evitar el desastre, como para responder cuando éste ocurra. La capacidad de respuesta al impacto de los desastres en el sector salud, especialmente en poblaciones vulnerables, debe ser una parte integral en la construcción de sistemas sólidos de salud pública. Se aplican las mismas consideraciones a los sistemas de cultivo y salud animal, por su enorme impacto sobre la seguridad alimenticia y la economía. Los gobiernos deben evaluar periódicamente qué tan preparados están en el ámbito de la salud pública regional, nacional e internacional.

c. Aplicaciones de la tecnología avanzada de la información. Las tecnologías de la información, incluyendo la geoespacial, son importantes para el monitoreo, identificación y alerta de desastres inminentes y en evaluación de su ubicación, naturaleza y alcance de los daños, número de muertes y de lesionados para poder enviar, coordinar y concentrar los esfuerzos de asistencia. Los países deben evaluar las posibles ventajas de los sistemas de tecnologías de la información especializados, para dar respuesta en casos de emergencia, en contraste con los sistemas compartidos que desempeñan 


\section{Ciencia y Educación (ISSN 2707-3378) \\ Vol. 1 No. 2 \\ Febrero del 2020}

múltiples funciones. De cualquier manera, la práctica sistemática (juegos de respuesta ante emergencias) con todos los jugadores clave, y los programas activos de participación pública y de educación, son fundamentales para el uso eficaz de estos sistemas.

d. Planeación, ingeniería e implementación de los estándares para minimizar la vulnerabilidad. Las pérdidas por desastres pueden disminuirse considerablemente mediante la mejora de los estándares de construcción de edificios, carreteras, sistemas eléctricos, sistemas de agua y otra infraestructura, y mediante la zonificación con objeto de reducir los índices de vulnerabilidad. Además de planear la protección de las poblaciones y la infraestructura moderna; también el patrimonio cultural y natural requieren protección ya que su pérdida es irreversible, es fundamental la investigación constante de diseños innovadores, de ingeniería y materiales, y la difusión de información sobre técnicas y materiales disponibles. Para poder ser eficaces, los gobiernos deben vigilar el fortalecimiento de los estándares.

e. Integración de la capacidad de resiliencia en los programas de asistencia al desarrollo. Los programas de asistencia al desarrollo pueden contribuir para que los países desarrollen su propia capacidad de resiliencia, a nivel local y nacional. Para que esto funcione, la asistencia debe llegar a quienes más la necesitan para poder reducir vulnerabilidades futuras. $\mathrm{La}$ educación pública y el compromiso, las lecciones aprendidas de desastres anteriores y la capacidad de comunicación, son especialmente importantes en el caso de las poblaciones y zonas vulnerables.
Dimensiones del comportamiento resiliente

Las dimensiones planteadas para la presente investigación son la aceptación y competencias personales:

a. Aceptación personal. La aceptación personal es fundamental para avanzar en el proceso de desarrollo integral y en el crecimiento espiritual. (Del Castillo, s.f.)

b. Competencias personales. Son las capacidades, conocimientos, destrezas y experiencias que hacen que una persona ejecute determinadas labores con eficiencia. Conjunto de conocimientos, habilidades $\mathrm{y}$ actitudes que posee una persona para desarrollar con normalidad las diferentes tareas a las que tiene que enfrentarse. (Rubio, 2010)

Por otro lado, García et. al. (2012, pág. 7) sugiere los siguientes indicadores del comportamiento resiliente:

a. Introspección. Hace mención de preguntarse a sí mismo y darse una auto respuesta honesta.

b. Independencia. Se define como la capacidad de establecer límites entre uno mismo y los ambientes adversos; alude a la capacidad de mantener distancia emocional y física, sin llegar a aislarse.

c. La capacidad de relacionarse. Inciden en la habilidad para establecer lazos íntimos y satisfactorios con otras personas. Aquí encontraríamos cualidades como empatía, la sociabilidad.

d. Iniciativa. Implica exigirse y ponerse a prueba en tareas progresivamente más exigentes. Se refiere a la capacidad de hacerse cargo de los problemas y de ejercer control sobre ellos. 


\section{Ciencia y Educación (ISSN 2707-3378) \\ Vol. 1 No. 2 \\ Febrero del 2020}

e. Humor. Alude al hecho de encontrar lo cómico en la tragedia. El humor ayuda a superar obstáculos y problemas, a hacer reír o reírse de lo absurdo de la vida.

f. Creatividad. Es la capacidad de crear orden, belleza y finalidad a partir del caos y el desorden. En la infancia se expresa con la creación de juegos, que son las vías para expresar la soledad, el miedo, la rabia y la desesperanza ante situaciones adversas.

g. Moralidad. Se refiere a la conciencia moral, a la capacidad de comprometerse de acuerdo con los valores sociales y de discriminar entre lo bueno y lo malo.

\section{Elementos para desarrollar la resiliencia}

En muchos casos, la resiliencia reforzada trae consigo múltiples beneficios, contribuyendo a disminuir el número de muertes, lesiones $\mathrm{y}$ pérdidas económicas ocasionadas por emergencias relativamente frecuentes al tiempo que se aumenta la resiliencia para enfrentar desastres futuros. Los elementos para desarrollar la resiliencia incluyen los siguientes (Academias G-Science, 2012, pág. 3):

a. Evaluación y monitoreo sistemáticos de los riesgos de desastres, la investigación continua para mejorar nuestra comprensión de las causas subyacentes, los sistemas mejorados de alerta y la concientización de los riesgos por parte de la sociedad y de todos los niveles de gobierno.

b. Establecimiento de una cultura e incentivos que fomenten la aceptación de responsabilidades de las comunidades, incluyendo el sector privado y organizaciones civiles para la planeación y cooperación en la preparación, respuesta y recuperación.

c. Planeación a largo plazo, inversión y fortalecimiento de medidas de mitigación o preventivas como el uso del suelo y otro código de zonificación y construcción.

d. Cooperación internacional en materia de planeación anticipada y respuesta rápida, así como de investigación y evaluación de los factores de riesgo.

\section{Conclusiones}

Con respecto a la revisión de la literatura, se sugiere una especificación más detallada de la resiliencia que describe las causas principales. La identificación de los principios de resiliencia se basa en accidentes (prácticas frágiles) y recuperación exitosa (prácticas resilientes). Los tres pasos para identificar los principios de resiliencia son:

- Identificar una cadena de eventos.

- Identificar las condiciones y la falta de condiciones.

- Identificar las restricciones subyacentes y las causas raíz.

Se identifican diferentes causas raíz en función de diferentes percepciones. Por lo tanto, diferentes enfoques pueden involucrar diferentes interpretaciones de resiliencia e identificar diferentes principios de resiliencia. Claramente, no hay consenso sobre la lista de principios de resiliencia. Por lo tanto, es importante que los principios se consideren como un conjunto, no como conceptos individuales independientes.

Academias G-Science (2012)describe dos de los principios básicos en la resiliencia: la capacidad de administrar los márgenes cerca de los límites de rendimiento y la capacidad de lograr una degradación y rebote con gracia y control de situaciones adversas en la gestión de riesgos. Estos principios incorporan cuestiones clave relacionadas con la resiliencia y su presencia en la literatura proporciona un grado de validación para este enfoque. 


\section{Ciencia y Educación (ISSN 2707-3378) \\ Vol. 1 No. 2 \\ Febrero del 2020}

Para especificar la resiliencia para mejorar la protección y la seguridad dentro de la unidad educativa, la clave es considerar los principios de resiliencia durante el desarrollo de los planes de gestión de riesgo y como parte de la gestión de la seguridad educativa. La perspectiva de resiliencia mejora la calidad de un análisis de riesgo. Esto se basa en tres argumentos.

- Se incrementa el alcance de incidentes pasados explorados en el análisis de riesgo; la comprensión de cómo evitar accidentes y mejorar la recuperación mejora la capacidad de recuperación y reduce el riesgo de futuros accidentes.

- Considerar los desafíos actuales en el análisis del riesgo futuro ayuda a hacer lo inesperado esperado, lo que lleva a un mayor enfoque en la degradación y recuperación con gracia. Teniendo en cuenta que la capacidad de recuperación y otras propiedades de resiliencia pueden aumentar la complejidad del sistema, lo que puede provocar accidentes. En consecuencia, para evitar un mayor riesgo, la resiliencia debe considerarse durante el análisis de riesgo al igual que otras acciones de mitigación.

- Existe un mayor enfoque en la gestión de los márgenes y las condiciones de los límites mediante el uso de indicadores proactivos; esto mejora la comprensión de los procesos clave que influyen en la seguridad.

Realizar análisis de riesgo con y sin considerar la resiliencia brinda la oportunidad de comparar perspectivas y acciones de mitigación e identificar diferencias. Según lo sugerido por Foré \& Grané, (2010), los resultados obtenidos deben medirse en términos del desempeño de seguridad de la unidad educativa.
Específicamente en los estudiantes de octavo grado.

Este estudio se enfoca en documentos revisados por pares para resaltar los determinantes de la resiliencia de los estudiantes de octavo grado en desastres y emergencias. Sin embargo, la falta de un estudio integrado llevó a esta revisión sistemática de la literatura; Dado que los niños pueden ser un grupo importante en un desastre, el concepto de resiliencia y la provisión de evidencia objetiva pueden hacer que las intervenciones sean más realistas y fructíferas.

Esto puede, quizás, conducir a cambios fundamentales en la planificación de los planes de gestión de riesgo en las unidades educativas logrando que sean más apropiado para su resiliencia en los estudiantes. Por lo tanto, este estudio puede ayudar a los planificadores y formuladores de políticas de gestión de riesgos de desastres y proveedores de atención de salud infantil, identificando la resiliencia y clarificando sus componentes que deben considerarse en las decisiones y planes futuros $\mathrm{y}$ tienen un efecto beneficioso en los niños y adolescentes en el futuro cercano. También se debe tener en cuenta que, si se considera la naturaleza cualitativa de este trabajo, la necesidad de un estudio cuantitativo y la provisión de una medición de la resiliencia es importante.

\section{Referencias bibliográficas}

Academias G-Science. (2012). Desarrollo de la resiliencia frente a desastres naturales $y$ tecnológicos. Academias G-Science: México.

Asociación Española para la Calidad. (2017). Asociación Española para la Calidad. Obtenido de Acción preventiva: https://www.aec.es/web/guest/centroconocimiento/accion-preventiva

Beltran, M. (13 de Febrero de 2013). Manejo de emociones. Obtenido 


\section{Ciencia y Educación (ISSN 2707-3378) \\ Vol. 1 No. 2 \\ Febrero del 2020}

http://tallerdecompetenciasdidacticas.blogsp ot.pe/2013/02/manejo-de-emocionesdefiniciones-y-citas.html

Centro Nacional de Estimación, Prevención y Reducción del Riesgo de Desastre. (2015). Manual para la evaluación de riesgos originados por Fenómenos Naturales. Lima: Centro Nacional de Estimación, Prevención y Reducción del Riesgo de Desastre.

Centro Nacional de Estimación, Prevención y Reducción del Riesgo de Desastres. (2014). Pautas para la incorporación de la Gestión del Riesgo de Desastres en el Reglamento de Organización y Funciones y en el Manual de Perfiles de Puestos de los Gobiernos Subnacionales. Lima: CENEPRED: Dirección de Fortalecimiento y Asistencia Técnica.

Chuquisengo, O. (2011). Guía de gestión de riesgos de desastres: Aplicación práctica. Lima: Soluciones Prácticas.

Comisión Económica para América Latina y el Caribe, Banco Interamericano de Desarrollo. (2000). Un tema del desarrollo: La reducción de la vulnerabilidad frente a los desastres. México: Comisión Económica para América Latina y el Caribe-Banco Interamericano de Desarrollo.

Del Castillo, H. (s.f.). La aceptación personal. Obtenido de http://es.catholic.net/op/articulos/58902/cat/ 224/la-aceptacion-personal.html

Foré, A., \& Grané, J. (2010). La resiliencia. Crecer desde la adversidad. Barcelona: Plataforma.

Fundación Nuestramente. (2013). Fundación Nuestramente. Obtenido de https://nuestramente.org/grupos/

García, M., Mateu, R., Flores, R., \& Gil, J. (2012). La resiliencia y las víctimas de desastres. Cuadernos de crisis y emergencias, 1-12.

Instituto Nacional de Defensa Civil. (15 de Julio de 2010). Terminología de defensa civil (5 ed.). Lima: Instituto Nacional de Defensa Civil (INDECI). Obtenido de http://bvpad.indeci.gob.pe/doc/pdf/esp/mat_ edu/terminologia2010.pdf
Lamas, H. (2002). Educar para la competencia personal. Palabra de Maestro, 11(35), 4447.

Lavell, A. (2003). La gestión local del riesgo: nociones y precisiones en torno al concepto y la práctica. Centro de Coordinación para la Prevención de los Desastres Naturales en América Central (CEPREDENAC). Programa de Naciones Unidas para el Desarrollo (PNUD).

Madrid, C. (24 de Marzo de 2015). Busqueda de Información pertinente.

Manciaux, M., Vanistendael, S., Lecomte, J., \& Cyrulnik, B. (2003). La resiliencia: estado de la cuestión.

Mateu, R., Gil, J., \& García-Renedo, M. (2009). ¿Hacia una escuela resiliente? Un estudio a través del profesorado de Educación Infantil y Primaria. España: (Tesis de maestría) UniversitatJaume I.

Ministerio de Educación. (2009). Gestión del riesgo en instituciones educativas. Lima: Ministerio de Educación.

Municipalidad de Guayaquil. (2011). Aprendamos: cómo actuar frente a desastres. Guayaquil: Municipalidad de Guayaquil.

Organización de las Naciones Unidas para la Educación, la Ciencia y la Cultura. (2011). Manual de gestión del riesgo de desastre para comunidadores sociales. Lima-Perú: Organización de las Naciones Unidas para la Educación, la Ciencia y la Cultura.

Perú. (08 de Febrero de 2011). Ley que crea el Sistema Nacional de Gestión del Riesgo de Desastres $N^{o}$ 29664. Obtenido de http://www.cenepred.gob.pe/web/es/data/pd f/Ley_SINAGERD.pdf

Rubio, P. (14 de Julio de 2010). Competencias personales. Obtenido de http://peprubio.blogspot.pe/2010/07/compet encias-personales.html

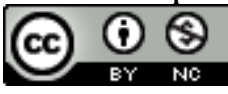
Creative Commons Reconocimiento-No Comercial 4.0 Internacional. Copyright (c) Marjorie Abigail Tapia Bajaña 